\title{
Aspectos ergonômicos e posturais e o trabalhador da área de saúde
}

\author{
Ergonomic and postural aspects and the health workers
}

\author{
Neusa Maria Costa Alexandre ${ }^{1}$
}

\begin{abstract}
Resumo
Os distúrbios osteomusculares representam um importante problema de saúde ocupacional. Os trabalhadores da área de saúde estão sujeitos a sintomas osteomusculares, atribuídos particularmente a condições ergonômicas inadequadas. Os procedimentos que envolvem a movimentação e o transporte de pacientes são considerados os mais penosos para esses trabalhadores, e a implementação de treinamento é parte obrigatória de programas de prevenção. O presente trabalho teve por objetivo sintetizar e descrever criticamente os pontos básicos das principais estratégias de prevenção e controle dos sintomas osteomusculares, em trabalhadores da área de saúde, dentro de uma abordagem ergonômica. A metodologia empregada foi uma revisão sistemática, articulando evidências empíricas produzidas por trabalhos científicos da autora. Nele se discutem temas relativos às orientações posturais, aspectos ergonômicos específicos e considerações sobre os procedimentos de movimentação e transporte de pacientes. Descreve também equipamentos que deveriam ser utilizados durante a movimentação e o transporte de pacientes considerando que eles devem ser urgentemente implementados nas atividades práticas da área de saúde.
\end{abstract}

Palavras-chave: Ergonomia. Postura. Dor lombar.

\begin{abstract}
Musculoskeletal disorders are an important occupational health problem. Health workers are at high risk of developing musculoskeletal symptoms due to inadequate ergonomic conditions. The procedures that involve the moving and transportation of patients are considered the most dangerous ones for health workers. Therefore, training programs on handling patients are a compulsory part of prevention programs. The purpose of the study was to synthesize and describe basic strategies to prevent and control musculoskeletal symptoms in health workers by means of an ergonomic theoretical framework. A systematic review was used in order to integrate empiric evidence produced by the author's scientific research. Themes related to postural orientations, specific ergonomic aspects and considerations about handling patients are presented. This study also describes the devices that should be used when moving and transferring patients, taking into consideration that they have to be urgently implemented in health practical activities.
\end{abstract}

Key words: Ergonomics. Posture. Low back pain.

\footnotetext{
1 Enfermeira. Professor Associado do Departamento de Enfermagem da Faculdade de Ciências Médicas da Universidade Estadual de Campinas (UNICAMP). Universidade Estadual de Campinas, Faculdade de Ciências Médicas, Departamento de Enfermagem. Cidade Universitária Zeferino Vaz, Barão Geraldo 13083-970 - Campinas, SP - Brasil. Caixa-Postal: 6111 - Telefone: (19) 3521 8820 Ramal: 8844 Fax: (19) 35218822.
} 


\section{Introdução}

Os distúrbios osteomusculares relacionados ao trabalho representam um sério dilema de saúde pública, por serem uma das mais importantes causas de incapacidade e absenteísmo. Entre essas afecções estão incluídas as patologias da coluna vertebral, e as dores lombares representam um risco para determinados grupos ocupacionais, dentre os quais se encontram os trabalhadores da área de saúde.

Organizações e pesquisadores de todo o mundo têm citado os trabalhadores da área de saúde como um grupo de risco em relação ao desenvolvimento de distúrbios osteomusculares relacionados ao trabalho, particularmente as algias vertebrais. Estudos têm mostrado ser elevada a ocorrência de sintomas musculoesqueléticos em múltiplas regiões corporais entre a equipe de enfermagem (ANDO et al., 2000; GURGUEIRA; ALEXANDRE; CORREA FILHO, 2003). No entanto, a dor lombar continua a ser o fator principal de absenteísmo, incapacidade funcional e procura por auxílio médico entre esses trabalhadores (COOPER; TATE; YASSI, 1998).

As agressões à coluna vertebral são causadas por inúmeros fatores inter-relacionados, entre os quais se salienta: as condições inadequadas de mobiliários e equipamentos; a manutenção de posturas estáticas e impróprias; a movimentação de pacientes acamados e o esforço físico ao transportá-los e os fatores organizacionais e psicossociais, tais como: equipe com número insuficiente, estresse, suporte social, satisfação e pressão no trabalho (CÉLIA; ALEXANDRE, 2004; PARADA; ALEXANDRE; BENATTI, 2002; RETSAS; PINIKAHANA, 2000; YIP, 2001). Considerando que as pesquisas das causas e o tratamento das dores lombares nesses trabalhadores têm gerado controvérsias, cada vez mais se buscam novas abordagens para enfrentar esse risco ocupacional. A literatura aponta estratégias como: implementação de medidas diagnósticas e preventivas precoces; orientações ergonômicas e posturais; utilização de dispositivos auxiliares; melhoria no ambiente de trabalho; revisão de aspectos organizacionais; programas de exercícios e alongamentos e alterações no estilo de vida (ALEXANDRE; SILVA; ROGANTE, 2001).

Dentro desse contexto, o presente estudo teve por objetivo sintetizar e descrever criticamente os pontos básicos das principais estratégias de prevenção e controle dos sintoma osteomusculares, em trabalhadores da área de saúde, dentro de uma abordagem ergonômica.

\section{Metodologia}

Trata-se de uma revisão sistemática, articulando evidências empíricas produzidas por trabalhos científicos da autora (RIERA; ABREU; CICONELLI, 2006). Para construção do estudo, considerou-se a inclusão de artigos publicados em revistas indexadas, com suporte de literatura internacional sobre o tema.

\section{Resultados}

Como resultado, foi possível descrever sinteticamente orientações básicas e inovadoras dentro do referencial teórico da ergonomia, englobando os equipamentos, as tarefas, o pessoal e o ambiente de trabalho.

\section{Avaliações e medidas para controle dos distúrbios} osteomusculares

Uma das primeiras medidas preventivas para evitar a cronicidade e a incapacidade para o trabalhado é uma avaliação precoce das afecções da coluna vertebral. Para isso, deve-se realizar uma avaliação cuidadosa, empregando métodos semiológicos padronizados. Uma propedêutica vertebral sistematizada deve englobar anamnese, com ênfase nos aspectos ocupacionais e ergonômicos, e exame físico específico, utilizando os seguintes métodos: inspeção estática e exame postural; inspeção dinâmica; palpação; avaliação da força e 
flexibilidade musculares; exame neurológico e manobras especiais (ALEXANDRE; MORAES, 2001).

É muito importante também avaliar e controlar aspectos dos sintomas osteomusculares tais como freqüiência e intensidade da dor; capacidade funcional e qualidade de vida dos pacientes e aspectos sociais e emocionais envolvidos. A literatura tem descrito escalas e questionários validados para avaliar essas diferentes dimensões (BARROS; ALEXANDRE, 2003; NORDIN; ALEXANDRE; CAMPELLO, 2003; VIGATTO; ALEXANDRE; CORREA FILHO, 2007). Esses instrumentos são muito importantes, pois fornecem dados para melhorar e avaliar a eficácia de tratamentos e programas de prevenção.

\section{Orientações ergonômicas e posturais}

Grande parte das agressões ao sistema osteomuscular em trabalhadores da saúde estão relacionadas a condições ergonômicas inadequadas de mobiliários, posto de trabalho e equipamentos utilizados nas atividades cotidianas, sendo as dores nas costas causadas por traumas crônicos repetitivos, que envolvem muitos outros fatores, além da manipulação de pacientes. Dessa forma, as recomendações acerca de um aspecto relevante do problema das algias osteomusculares, que é a prevenção e controle, têm caminhado em direção a uma abordagem ergonômica mais ampla (ALEXANDRE, 1998; ALEXANDRE; MORAES, 1998; BUCKLE, 2005; OWEN, 2001).

Assim, é importante salientar as seguintes recomendações:

- Orientações em relação às técnicas adequadas de levantamento, manuseio e transporte de cargas

- Avaliar a carga.

- Manter um espaço livre para acesso à carga.

- Obter condições seguras do solo e do trajeto a ser percorrido.

- Posicionar os pés corretamente.
- Segurar a carga usando totalmente as mãos.

- Levantar cargas do chão com o dorso retificado e os joelhos dobrados.

- Carregar a carga o mais próximo possível do corpo.

- Evitar movimentos de torção em torno de eixo vertical do corpo.

- Utilizar, sempre que possível, elementos e equipamentos auxiliares.

- Participar periodicamente de programas de treinamento.

- Orientações em relação à postura e movimentos

- Proporcionar variação de posições e atividades.

- Observar a altura da bancada de trabalho, de acordo com o tamanho do trabalhador e o tipo de serviço. executado ( bancadas para preparar medicações, medir crianças, coletar sangue).

- Manter altura de bancada ajustável, quando utilizada por diferentes pessoas (camas, macas).

- Manter espaço suficiente para os membros inferiores.

- Colocar os pés alternadamente em um banquinho ao ter que trabalhar em pé por tempo prolongado.

- Evitar a inclinação do tronco mantendo os membros inferiores esticados (ex. ao retirar material esterilizado de armário).

- Evitar alcances excessivos.

- Evitar o alongamento excessivo da coluna vertebral.

- Armazenar objetos pesados dentro de uma amplitude de alturas próximas à cintura e os objetos leves em qualquer altura situada entre 
o joelho e o ombro (caixa de instrumental, monitores, roupas, soros, etc).

- Colocar materiais em um nível que nunca ultrapasse a altura da cabeça.

- Utilizar uma escadinha ao retirar objetos de partes altas de estantes já construídas (ex. pegar soros em armários) .

- Orientações em relação aos equipamentos

- Evitar a utilização de mobiliários e equipamentos improvisados.

- Atentar para uma manutenção adequada e periódica dos equipamentos hospitalares..

- Modernizar o trabalho do pessoal de enfermagem pelo uso de equipamentos modernos, dando-se mais atenção ao provisionamento de auxílios mecânicos.

- Avaliar equipamentos e mobiliários hospitalares, levando em conta fatores ergonômicos.

- Verificar localização e orientação de dispositivos e comandos.

- Orientações organizacionais e psicossociais

- Proporcionar variação de tarefas e atividades.

- Evitar movimentos repetitivos.

- Verificar conforto térmico, visual e acústico no ambiente de trabalho.

- Avaliar jornada de trabalho e distribuição de períodos de pausas.

- Implantar modificações que garantam aos trabalhadores mais criatividade, autonomia e satisfação.

- Proporcionar número suficiente de trabalhadores por turno.
Orientações em relação à movimentação $e$ transporte de clientes

Como os procedimentos que envolvem a movimentação e o transporte de pacientes são considerados os mais penosos e perigosos para os trabalhadores da saúde, a implementação de treinamentos e reciclagem é parte obrigatória dos programas de prevenção de lesões osteomusculares.. Tais procedimentos devem ser aprendidos e praticados de forma planejada e sistemática (ALEXANDRE; ROGANTE, 2000) e seu ensino deve ser acompanhado por uma avaliação do local de trabalho e apresentar alternativas para torná-los menos prejudiciais (RADOVANOVIC; ALEXANDRE, 2002). As habilidades em movimentação de pacientes devem ser complementadas com o estabelecimento de práticas seguras de trabalho, dentro de uma estrutura ergonômica, e, sempre que possível, deve-se usar materiais e equipamentos auxiliares Um cuidadoso planejamento, antes de se iniciar esses procedimentos, é essencial e imprescindível. Dessa forma, desenvolveram-se orientações básicas com um enfoque ergonômico.

Considerando-se tais aspectos, dividiu-se essa fase em quatro partes :

- Avaliação das condições e preparo do cliente

Inicialmente, deve-se fazer uma avaliação das condições físicas da pessoa que será movimentada, de sua capacidade de colaborar, bem como a observação da presença de soros, sondas e outros equipamentos instalados. Também é importante, para um planejamento cuidadoso do procedimento, uma explicação, ao paciente, do modo como se pretende movê-lo, como pode cooperar, para onde será encaminhado e qual o motivo da locomoção. Vale a pena salientar que o cliente deve ser orientado a ajudar, sempre que for possível, que não deve ser mudado rapidamente de posição e tem que usar chinelos ou sapatos com sola antiderrapante. Outro 
ponto muito importante é que a movimentação e o transporte de obesos precisam ser minuciosamente avaliados e planejados, e cabe usar, sempre que possível, auxílios mecânicos.

- Preparo do ambiente e dos equipamentos

Considerando-se que determinados aspectos ergonômicos do posto de trabalho podem prejudicar atividades ocupacionais, tais como os procedimentos relacionados com movimentação e transporte, expõem-se, a seguir, os principais cuidados que necessitam ser observados:

- Verificar se o espaço físico é adequado para não restringir os movimentos.

- Examinar o local e remover os obstáculos.

- Observar a disposição do mobiliário.

- Obter condições seguras com relação ao piso.

- Colocar o suporte de soro ao lado da cama, quando necessário.

- Elevar ou abaixar a altura da cama, para ficar no mesmo nível da maca.

- Travar as rodas da cama, maca e cadeira de rodas ou solicitar auxílio adicional.

- Adaptar a altura da cama ao trabalhador e ao tipo de procedimento que será realizado.

Deve-se, também, utilizar equipamentos auxiliares e adaptar as condições do ambiente a cada paciente em particular. Nesse caso, pode ser necessário:

- Colocar barras de apoio em banheiros.

- Elevar a altura do vaso sanitário (compensadores de altura para vasos convencionais)..

- Utilizar cadeira de rodas própria para banho ou higiene.
- Preparo da equipe

Algumas orientações, especificamente relacionadas com os princípios básicos de mecânica corporal, devem ser utilizadas pelo pessoal de enfermagem, durante a manipulação de pacientes:

- Deixar os pés afastados e totalmente apoiados no chão.

- Trabalhar com segurança e com calma.

- Manter as costas eretas..

- Usar o peso corporal como um contrapeso ao do paciente.

- Flexionar os joelhos, ao invés de curvar a coluna.

- Abaixar a cabeceira da cama ao mover um paciente para cima.

- Utilizar movimentos sincrônicos.

- Trabalhar o mais próximo possível do corpo do cliente, que deverá ser erguido ou movido..

- Usar uniforme que permita liberdade de movimentos e sapatos apropriados.

- Realizar a manipulação de pacientes com a ajuda de, pelo menos, duas pessoas.

- Movimentação e transporte de clientes

Lembrar que o paciente deve ser estimulado a movimentar-se de forma independente, sempre que não existir contra-indicações nesse sentido. Outro ponto que não pode ser esquecido é procurar ter à disposição camas e cadeiras apropriadas, dependendo das condições e necessidades do cliente. $O$ ideal são camas com altura regulável, que possa ser ajustada de acordo com o procedimento que será realizado. Durante esses procedimentos, deve-se, sempre que possível, utilizar elementos auxiliares, tais como: barra tipo trapézio no leito, plástico antiderrapante para os pés, plástico facilitador de movimentos, cintos e pranchas de transferência, discos giratórios e auxílios mecânicos, entre outros. 
Os principais motivos que levam os trabalhadores de saúde a movimentar e transportar os clientes são: colocar ou retirar comadres, movimentar para um dos lados da cama, colocar em decúbito lateral, movimentar para a cabeceira da cama, auxiliá-los a levantarem-se de cadeira ou poltrona, auxiliá-los a deambularem, transferir do leito para uma poltrona ou cadeira de rodas, transferir do leito para uma maca, entre outros. Para executar cada um desses procedimentos, existem orientações específicas, com enfoque ergonômico, que devem ser ajustadas a cada situação. Publicações descrevem em pormenores os procedimentos que envolvem a movimentação e o transporte de pacientes (ALEXANDRE; ROGANTE, 2000).

Equipamentos que auxiliam na manipulação de clientes

A literatura tem demonstrado que existem inúmeros dispositivos auxiliares que precisam ser utilizados e divulgados (ALEXANDRE; SILVA; ROGANTE, 2001; SILVA; ALEXANDRE, 2002). Programas ergonômicos que utilizam materiais auxiliares na manipulação de pacientes têm comprovado redução significativa nos problemas de coluna vertebral em trabalhadores de enfermagem. Estudos biomecânicos reforçam a necessidade da utilização de equipamentos especiais na transferência de pacientes dependentes, pois comprovam que esses equipamentos diminuem significativamente as forças compressivas sobre as costas, durante a execução dessas transferências (ULIN et al., 1997; ZHUANG et al., 1999).
Nesta fase do estudo, procura-se fazer uma breve descrição desses dispositivos que, por uma questão didática, foram divididos em: auxílio à movimentação, à transferência e às atividades de higiene e de locomoção.

- Auxílio à movimentação de pacientes no leito

Barra tipo trapézio no leito: tem como objetivo auxiliar o paciente a sentar-se, mover-se para baixo ou para cima e elevar o quadril no leito de forma independente. É constituída de uma estrutura fixa ou móvel, com uma cinta para regulagem de altura e um triângulo para apoio das mãos.

Camas com altura ajustável: possuem um sistema de regulagem de altura e movimentações de acordo com o tipo de procedimento a ser realizado e com a altura do trabalhador. Pode ser elétrica ou hidráulica (mecânica), sendo sua estrutura em aço inoxidável ou em aço revestido de material antioxidante. Estudos ergonômicos têm demonstrado que camas com alturas ajustáveis facilitam a transferência de pacientes e diminuem as forças no segmento L5-S1 da coluna vertebral (CABOOR et al., 2000).

Plásticos facilitadores de movimentos: são estruturas tubulares com superfícies deslizantes, de diferentes tipos e tamanhos. Auxiliam as mudanças de decúbito e na movimentação do paciente para cima ou para baixo no leito. (Figuras 1 e 2).

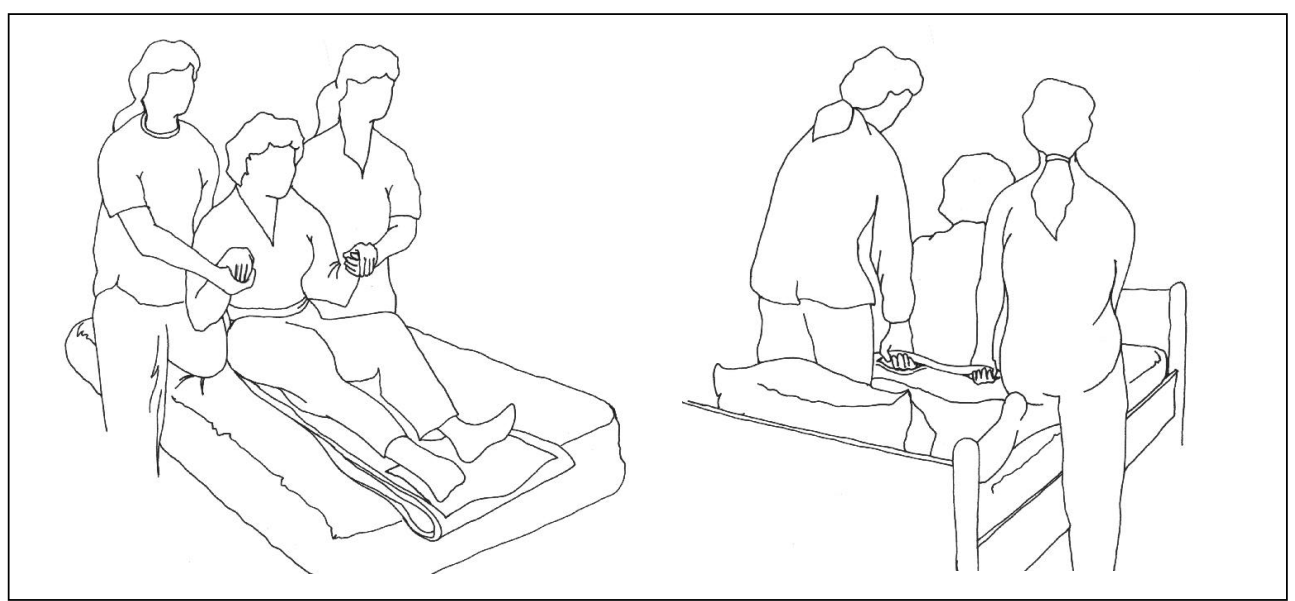

Figuras 1 e 2. Movimentar pacientes no leito utilizando plásticos facilitadores de movimentos. 
Plástico antiderrapante para os pés: é utilizado como apoio para os pés no leito, com o objetivo de facilitar a movimentação independente do paciente para cima no leito.

Blocos de mão: são dispositivos em forma de bloco, com base antiderrapante e anatômicos para suporte das mãos. Servem para auxiliar o paciente a movimentar-se no leito na posição sentada, de uma forma independente.

Escada de cordas: auxilia o paciente a sentarse de forma independente. É constituída de tubos de material rígido, de aproximadamente $20 \mathrm{~cm}$ de comprimento e $25 \mathrm{~mm}$ de diâmetro, fixados entre si nas extremidades com corda. A escada fica fixada ao pé da cama com o primeiro degrau ao alcance do paciente. (Figura 3)

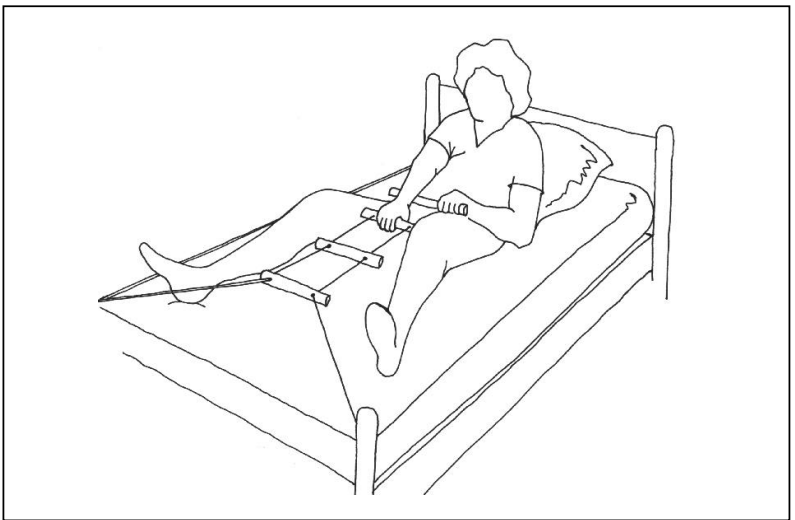

Figura 3. Paciente sentando com a ajuda de uma escada de cordas.

- Auxílio à transferência de pacientes

Cintos de transferência: são cintos ajustáveis à cintura do cliente, confeccionados com materiais resistentes como lona ou juta. Possuem alças verticais e horizontais que servem de apoio ao auxiliar na transferência e na deambulação de pacientes.

Tábuas de transferência: pranchas de plástico rígido ou de madeira, de diferentes tamanhos e formatos. São utilizadas para ajudar a transferência do paciente do leito para a cadeira de rodas ou para a poltrona, exigindo dele bom controle manual e força na parte superior do corpo. Esses equipamentos são baratos e fáceis de transportar, mas exigem que as superfícies de transferências tenham a mesma altura. (Figuras 4 e 5)

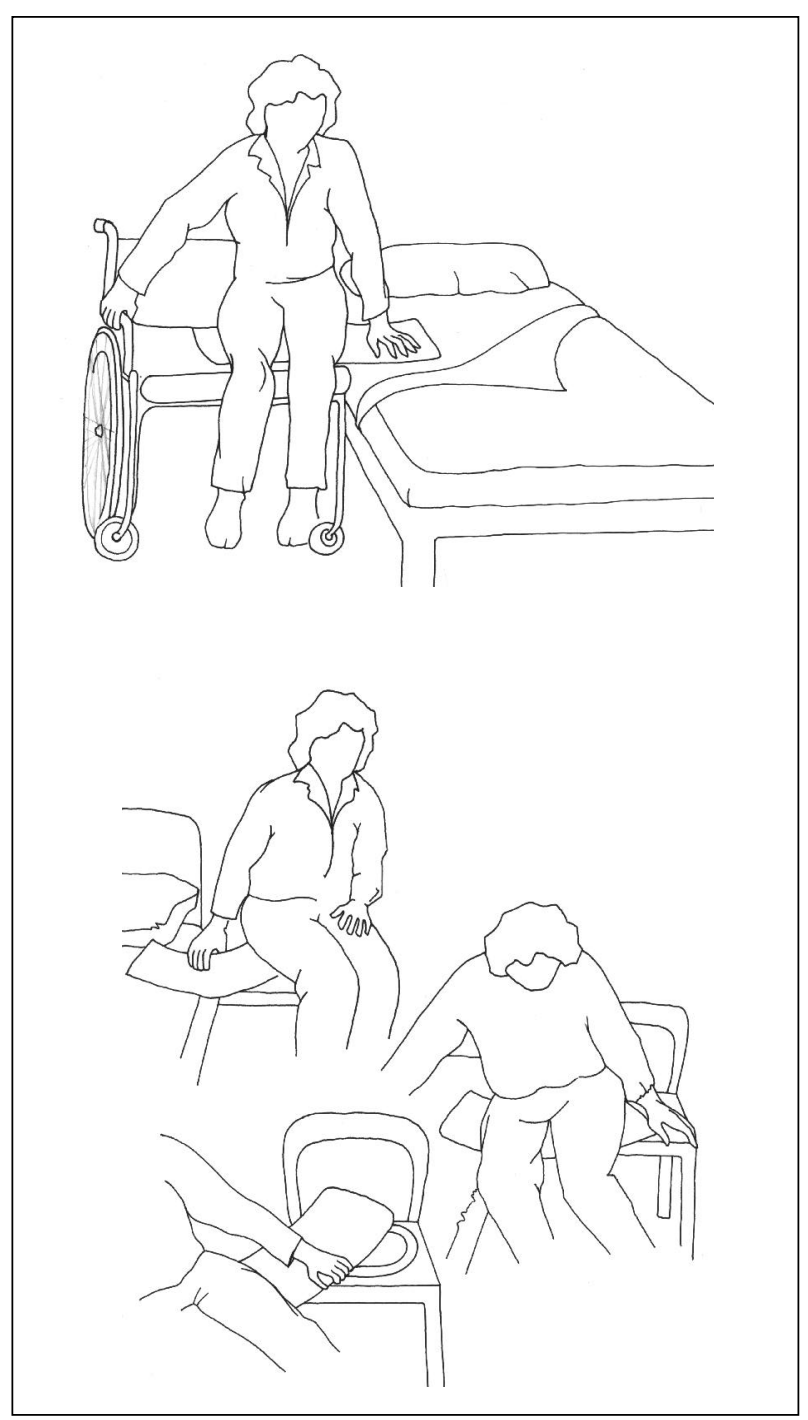

Figuras 4 e 5. Transferência de pacientes com o uso de pranchas.

Discos giratórios para os pés: são compostos por dois discos de plástico que giram entre si, sendo a face inferior confeccionada de material antiderrapante. $\mathrm{O}$ cliente coloca seus pés no disco, o que facilita sua rotação em 90 graus.

Pranchas de transferências para macas: são dispositivos em forma de prancha ou rolo, envoltos em plásticos deslizantes, com a finalidade de 
transferir o paciente da maca para a cama e viceversa. São indicadas para transferências em mesa cirúrgica, ambulâncias, macas, entre outras.

Elevadores de transferência: são dispositivos utilizados para as atividades em que a transferência para o banho e outros procedimentos impliquem risco para a segurança do paciente e para o trabalhador de saúde. Encontram-se vários modelos desses equipamentos, e eles permitem de levantar pacientes dependentes e transferí-los para cadeiras de rodas, vasos sanitários, banheiras, etc. Possuem sistema de regulagem da altura e de movimentação de acordo com o tipo de procedimento a ser realizado e a altura do trabalhador. Podem ser elétricos ou hidráulicos, sua estrutura é confeccionada em aço inoxidável ou aço revestido em material antioxidante. São acompanhados de acessórios como: suportes e cintas de apoio para quadril , região dorsal e membros inferiores.

- Auxílio às atividades de higiene

Cadeira de rodas para banho, cadeira higiênica e banquetas para banho: esses equipamentos são utilizados nas atividades de higiene corporal, proporcionando mais conforto e segurança ao paciente. Possuem estrutura de material plástico, alumínio ou aço inoxidável, e apresentam diversas características ergonômicas, como ajuste ao vaso sanitário e a banheiras; suporte para o apoio dos braços e das mãos; encosto ajustável; assento rotatório; rodas opcionais e assento perfurado anatomicamente para facilitar a higiene íntima (CORNÉLIO; ALEXANDRE, 2005).

Barras de apoio na parede e suporte de segurança para vaso sanitário: apresentam formas e desenhos em função das necessidades dos usuários e do ambiente. São construídas de aço inoxidável, em diversos tamanhos, com diâmetro aproximado de $32 \mathrm{~mm}$, superfície de agarre com frisos antideslizantes, e favorecem a segurança e a adaptação das mãos.

Compensadores de altura para vaso sanitário: são dispositivos de plástico, com alturas variáveis e com mecanismos de segurança como presilhas, para serem fixados em vaso sanitário convencional, de formato anatômico. Servem para auxiliar o paciente a se sentar com segurança e mais facilidade no vaso sanitário, e a se levantar dele. Existem no mercado diversos modelos, tais como com frente vazada, permitindo melhor acesso ao asseio perineal, e com suporte lateral para apoio das mãos.

- Auxílio às atividades de locomoção

Andadores, muletas e bengalas: são suportes, com alturas ajustáveis, de madeira ou de alumínio, leves e com pés de borracha antiderrapante. Servem de apoio a pessoas com limitação de movimentos e facilitam a locomoção. Existem diversos modelos no mercado para atender, de forma adequada, todos os tipos de incapacidade de locomoção do paciente.

Cadeiras de roda: há grande diversidade no mercado, com possibilidade de adequar o modelo à necessidade do paciente e do ambiente. Devem ter travas nas rodas, braços e peseiras removíveis que são itens para a segurança do paciente. A altura ajustável é um ponto importante para facilitar a locomoção dos pacientes de forma independente (JORGE; ALEXANDRE, 2005).

\section{Orientações gerais}

Considerando que os estudos sobre as dores musculoesqueléticas têm gerado inúmeras controvérsias e que geralmente abrangem múltiplas variáveis com complexo inter-relacionamento, cada vez mais se buscam novas abordagens para enfrentar e aliviar os sintomas, que englobam as seguintes orientações (NORDIN; ALEXANDRE; CAMPELLO, 1997):

- Praticar atividades físicas regularmente

- Realizar exercícios e alongamentos específicos para a coluna vertebral

- Evitar a obesidade e o tabagismo 
- Utilizar posições, colchões e travesseiros adequados para dormir

- Realizar relaxamento

- Utilizar massagem

- Aplicar calor no local da dor

\section{Considerações Finais}

Países desenvolvidos têm elaborado e colocado em ação políticas administrativas e legais que levam em conta o tratamento e a prevenção das dores lombares em trabalhadores da área da saúde. Essas medidas englobam inúmeras estratégias com uma abordagem ergonômica que precisam urgentemente serem implementadas na realidade brasileira. $\mathrm{O}$ presente trabalho procurou expor, de forma resumida, essas medidas de controle e prevenção dos sintomas osteomusculares.

\section{Agradecimentos}

Ao Conselho Nacional de Desenvolvimento Científico e Tecnológico (CNPq).

\section{Referências}

ALEXANDRE, N. M. C. Ergonomia e as atividades ocupacionais de enfermagem. Revista da Escola de Enfermagem da USP, São Paulo, v.32, n.1, p.84-90, 1998.

. Evaluation of a program to reduce back pain in nursing personnel. Revista de Saúde Pública, São Paulo, v.35, n.4, p.356-61, 2001.

ALEXANDRE, N. M. C.; MORAES, M. A. A. Modelo de avaliação físico-funcional da coluna vertebral. Revista Latino-Americana de Enfermagem, Ribeirão Preto, v.9, n.2, p.67-75, 2001.

Proposta educativa com enfoque ergonômico para auxiliar na prevenção de lesões musculoesqueléticas na equipe de enfermagem. Revista Brasileira de Enfermagem., Brasília, v.51, n.4, p.629-42, 1998.

ALEXANDRE, N. M. C.; ROGANTE, M. M. Movimentação e transferência de pacientes: aspectos ergonômicos e posturais. Revista da Escola de Enfermagem da USP, São Paulo, v.34, n.2, p.165-73, 2000.
ALEXANDRE, N. M. C.; SILVA, F. B.; ROGANTE, M. M. Aparatos utilizados em la movilización de pacientes: um enfoque ergonômico. Temas de Enfermería Actualizados, v.43, n.9, p.19-23, 2001

ANDO, S.; ONO, Y.; SHIMAOKA, M.; HIRUTA, S.; HATTORI, Y.; HORI, F.; TAKEUCHI, Y. Associations of self estimated workloads with musculoskeletal symptoms among hospital nurses. Journal of Occupational and Environmental Medicine., Baltimore, v.57, n.3, p.211-16, 2000.

BARROS, E. N. C.; ALEXANDRE, N. M. C. Cross-cultural adaptation of the Nordic musculoskeletal questionnaire. International Nursing Review, Geneva, v.50, n.2, p.101-7, 2003.

BUCKLE, P. Ergonomics and musculoskeletal disorders: overview. Occupational medicine., London, v.55, n.3, p.164-7, 2005.

CABOOR, D. E.; VERLINDEN, M. O.; ZINZEN, E.; VAN ROY, P.; VAN RIEL, M. P.; CLARYS, J. P. Implications of an adjustable bed height during standard nursing tasks on spinal motion, perceived exertion and muscular activity. Ergonomics, London, v.43, n.10, p.1771-80, 2000.

CÉLIA, R. C. R. S.; ALEXANDRE, N. M. C. Aspectos ergonômicos e sintomas osteomusculares em um setor de transporte de pacientes. Revista Gaúcha de Enfermagem., Porto Alegre, v.25, n.1, p.33-43, 2004.

CORNÉLIO, M. E.; ALEXANDRE, N. M. C. Avaliação de uma cadeira de banho utilizada em ambiente hospitalar: Uma abordagem ergonômica. Revista Brasileira de Enfermagem, Brasília, v.58, p.405-10, 2005.

COOPER, J. E.; TATE, R. B.; YASSI, A. Components of initial and residual disability after back injury in nurses. Spine, v.23, n. 19, p. 2118-22, 1998.

GURGUEIRA, G. P.; ALEXANDRE, N. M. C.; CORREA FILHO, H. R. Prevalência de sintomas músculoesqueléticos em trabalhadores de enfermagem. Revista Latino-Americana de Enfermagem, Ribeirão Preto, v.11, n.5, p.608-613, 2003.

JORGE, S. S.; ALEXANDRE, N. M. C. Avaliação ergonômica de cadeira de rodas utilizada no transporte de pacientes em hospital. Revista enfermagem UERJ, Rio de Janeiro, v.13, n.2, p.181-187, 2005.

NORDIN, M.; ALEXANDRE, N. M. C.; CAMPELLO, M. Measures for low back pain: a proposal for clinical use. Revista Latino-Americana de Enfermagem, Ribeirão Preto, v.11, n.2, p.52-5, 2003.

NORDIN, M.; ANDERSON, G. B. J.; POPE, M. H. (Ed.). Musculoskeletal disorders in the workplace. St Louis: Mosby; 1997. 
OWEN,B. D. Preventing injuries using an ergonomic approach. AORN Journal, Denver, v.72, n.6, p.1031-6, 2001.

PARADA, E. O.; ALEXANDRE N, M. C.; BENATTI, M. C. C. Lesões ocupacionais afetando a coluna vertebral em trabalhadores de enfermagem., Revista Latino-Americana de Enfermagem, Ribeirão Preto, v.10, n.1, p.64-9, 2002.

RADOVANOVIC, C. A. T.; ALEXANDRE, N. M. C. Desenvolvimento de um instrumento para avaliar a movimentação e transferência de clientes: um enfoque ergonômico. Revista da Escola de Enfermagem da USP, São Paulo, v.36, n.3, p.231-9, 2002.

RETSAS, A.; PINIKAHANA, J. Manual handling activities and injuries among nurses. Journal of Advanced Nursing, Oxford, v.31, n.4, p.875-83, 2000.

RIERA, R.; ABREU, M. M.; CICONELLI, R. M. Revisões sistemáticas e metanálises na reumatologia. Revista Brasileira de Reumatologia, São Paulo, v.46, supl.1, p.811,2006.

SILVA, F. B.; ALEXANDRE, N. M. C. Presença e utilização de equipamentos para movimentação e transporte de pacientes em um hospital universitário. Revista Paulista de Enfermagem, São Paulo, v.21, p.255-61, 2002.
ULIN, S. S.; CHAFFIN, D. B.; PATELLOS, C. L.; BLITZ, S. G.; EMERICK, C. A.; LUNDY, F.; MISHER, L. A biomechanical analysis of methods used for transferring totally dependent patients. SCI Nursing, New York, v.14, n.1, p.19-26, 1997.

VIGATTO, R.; ALEXANDRE, N. M. C.; CORREA FILHO, H. R. Development of a Brazilian Portuguese version of the Oswestry Disability Index. Cross-cultural adaptation, reliability, and validity. Spine, Philadelphia, v.32, n.4, p.481$6,2007$.

YIP, Y. B. A study of work stress, patient handling activities and the risk of low back pain among nurses in Hong Kong. Journal of Advanced Nursing, Oxford, v.36, n.6, p.794804,2001

ZHUANG, Z;; STOBBE, T. J.; HSIAO, H.; COLLINS, J. W.; HOBBS, G. R. Biomechanical evaluation of assistive devices for transferring residents. Applied Ergonomics, Guildford, v.30, n.4, p.285-294, 1999. 